\title{
CMY3
}

\section{Reconfigurable Wavelength-Selective Reflector Consisting of Coupled Polymeric Microring Resonators}

\author{
Jacob Scheuer \\ Center for the Physics of Information, Applied Physics, California Institute of Technology, MC 128-95, Pasadena, CA 91125, USA \\ kobvacaltech.edn \\ George T. Paloczi and Amnon Yariv \\ Department of Applied Physics, California Institute of Technology, MC 128-95, Pasadena, CA 91125, USA
}

Cheng Zhang

Pacific Wave Industries, Inc., I29 Sheldon St. El Segundo, California 90245

Larry R. Dalton

Department of Chemistry, University of Washington, Seattle, Washington 98195, USA

\begin{abstract}
We propose and demonstrate a novel reconfigurable wavelength-selective reflector for planar lightwave technology based on coupled microring resonators. Narrow-band reflection and strong sidelobe suppression are demonstrated in an optical polymer device using the Vernier effect. (C)2005 Optical Society of America

OCIS codes: 230.5750 Resonators; 160.5470 Polymers
\end{abstract}

\section{Introduction}

Lasers that exhibit stable wavelength and narrow bandwidth are key elements for modern optical communication systems [1]. For wavelength division multiplexing (WDM) applications, it is also desired that the laser wavelength be reconfigurable or trimable. In most lasers, the demand for stable lasing wavelength is fulfilled by employing either Bragg reflectors [2] or external cavities [3-4]. However, employment of DBRs or external cavities requires additional high resolution processing steps that make the fabrication of the structure more difficult. In addition, tuning the reflection wavelength of a DBR over wide range without deteriorating the reflection characteristics is not simple and requires careful design and implementation. The integration of reflectors into planar optical circuits is highly advantageous, enabling compact reflectors, realization with a wide variety of materials, and integration with additional on-chip photonic devices such as modulators, couplers, sources, etc.

Here, we present and demonstrate a novel geometry for an in-plane, wavelength-selective reflector in the spirit of [5], which is based on coupled microring resonators. The utilization of optical polymers for the device enables a simple fabrication procedure. In addition, the usage of multiple microrings allows for improved sidelobe suppression and tunability.

\section{Design concept and theory}

Figure 1 shows an optical image of the device. Light is launched into to input waveguide and equally divided between the two arms of the $\mathrm{Y}$-junction. The signal in each arm is filtered twice by passing through the cascaded microring add/drop filters. The transfer function of the composite filter is given by:

$$
D(\omega)=\frac{k_{1} k_{2}\left(1-L_{1}\right)^{1 / 4}\left(1-L_{2}\right)^{1 / 4} e^{i \pi R_{1} n_{e f f} \omega} e^{i \pi R_{2} n_{e f f} \omega}}{\left[1-\left(1-k_{1}\right) \sqrt{1-L_{1}} e^{i 2 \pi R_{1} n_{e f f} \omega}\right] \cdot\left[1-\left(1-k_{2}\right) \sqrt{1-L_{2}} e^{i 2 \pi R_{2} n_{e f f} \omega}\right]}
$$

where $k_{i}, L_{i}$ and $R_{i}$ are respectively the power coupling, loss per revolution and the radius of the $i$ ring $(i=1,2)$. Note that the transfer function (1) is independent of the order of the two rings and, therefore, the light in both arms experiences identical filtering. Finally the two parts of the filtered signal are combined by the Y-splitter to generate the reflected signal. The remaining signal, which is not within the bandwidth of the filter, continues through ports 2 and 3 (see Fig. 1) and can be used as a monitor for tuning the resonance wavelengths of the microrings

The architecture of the device shown in figure 1 exhibits several inherent advantages. The reflected wavelengths must be resonant for all resonators composing the filter, thus allowing for the reflection of a small set of wavelengths (or even a single wavelength) by using microrings with different radii (the Vernier effect). 


\section{CMY3}

Moreover, the device structure ensures that the two parts of the signal experience, literarily, identical paths and thus, no post-fabrication balancing of the device is required.

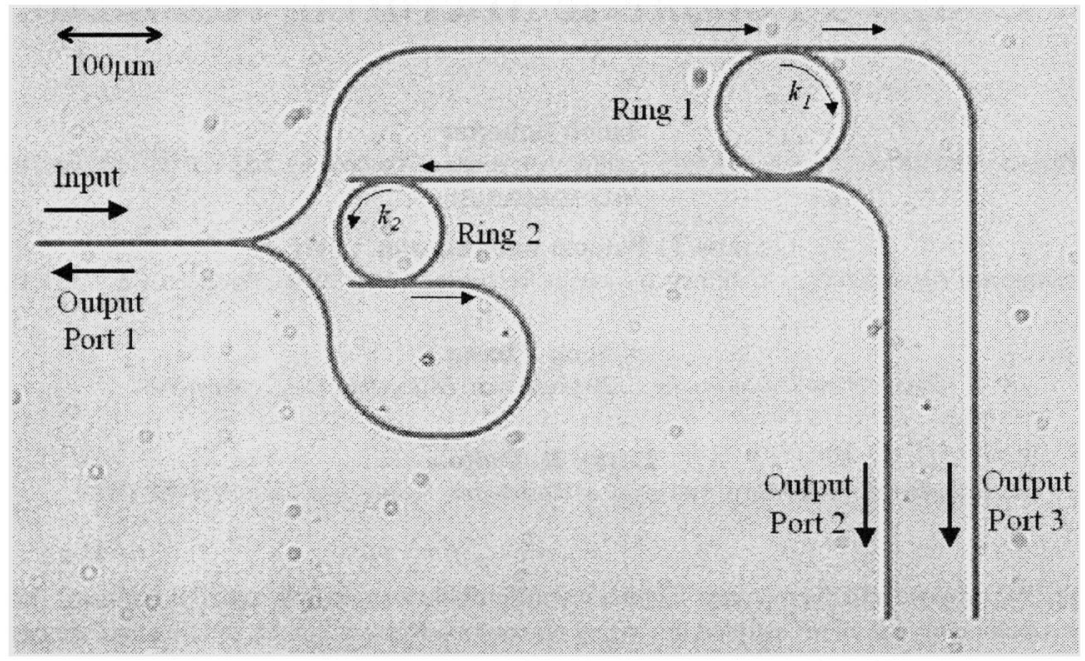

Fig. 1. An optical image of the proposed wavelength-selective reflector

Figure 2 shows the calculated spectral response of the three ports for a device consisting of microrings with radii $R_{1}=125 \mu \mathrm{m}, R_{2}=100 \mu \mathrm{m}, k_{1}=k_{2}=0.3, L_{1}=0.3, L_{2}=0.25$ (corresponding to loss of $20 \mathrm{~dB} / \mathrm{cm}$ ) and group index of $n_{g}=1.655$. The corresponding free spectral ranges (FSR) of the resonators are $\mathrm{FSR}_{1}=230.8 \mathrm{GHz}$ and $\mathrm{FSR}_{2}=288.5 \mathrm{GHz}$. Because of the $4: 3$ ratio between the resonators radii, every fourth resonance of ring 2 aligns with a resonance of ring 1, generating an effective (collective) FSR of $1154 \mathrm{GHz}$. This can be clearly seen in figures 2(a) and 2(b) showing the reflected (port 1) and transmitted (port 2) spectra.
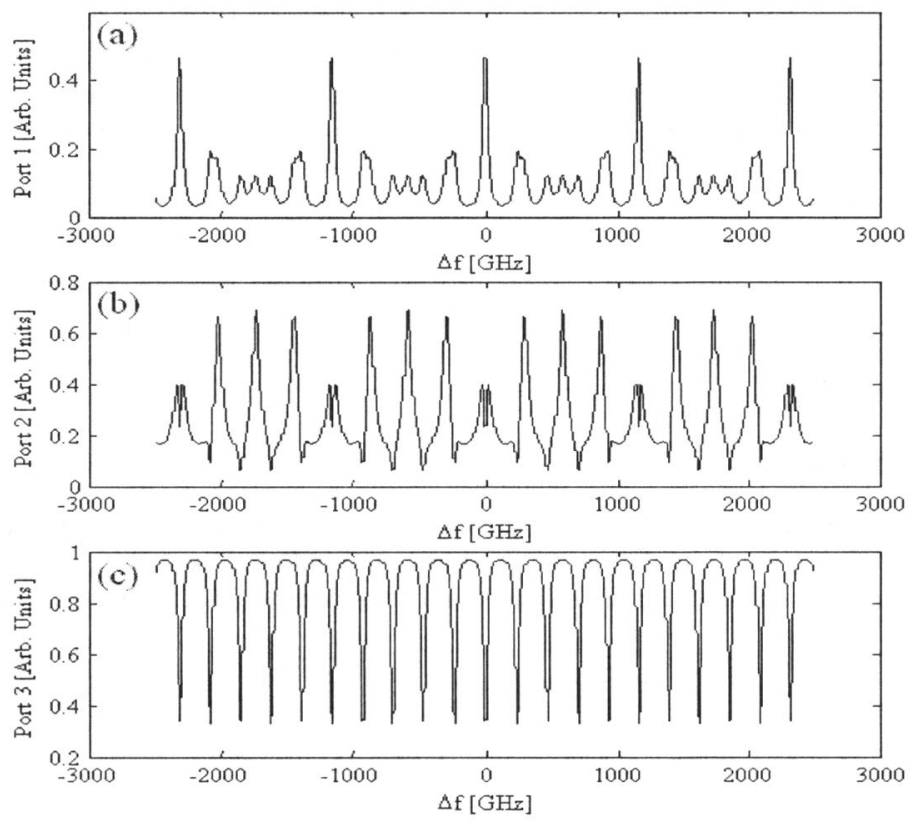

Fig. 2. An optical image of the proposed wavelength-selective reflector

\section{Fabrication and measurement}

Devices were fabricated using the soft-lithography replica-molding method [6, 7] of a polymer doped with electro-optic chromophore CLD-1 [8]. The radii of the microrings were $R_{l}=125 \mu \mathrm{m}, R_{2}=100 \mu \mathrm{m}$, the waveguide dimensions were approximately $1.6 \mu \mathrm{m} X 2 \mu \mathrm{mm}$, and the refractive index $\sim 1.6 @ 1550 \mathrm{~nm}$. The lower and upper claddings were respectively thermally grown silicon oxide $(\sim 1.45 @ 1550 \mathrm{~nm})$ and air. The group index of this 


\section{CMY3}

waveguide structure was calculated using finite-difference mode solver software and was found to be $\sim 1.655$. This number was verified by measuring the FSR of Ring 1 using port 3.

Figure 3(a) depicts the reflected spectrum measured at the output of port 1 . The good agreement between the measured reflection and the theory (see Fig. 2(a)) is evident: On the two sides of each mutual resonance frequencies of both rings there are lower reflection peaks. The region between these triplets is occupied by small oscillations. The measured intensity ratio between the amplitudes of the main reflection peaks and those of the satellite lobes (Fig. 3(a)) is $\sim 1.67$ which is in good agreement with the predicted ratio of 1.75 . Figure $3(\mathrm{~b}$ ) shows the tuning of on of the main reflection peaks (marked by the dashed rectangular) by photo-bleaching of the chromophores in ring 1 (Fig. 1) using white light source. Photo bleaching ring 1 for approximately 45 seconds, shifted of resonance frequencies of the ring by $\sim 0.46 \mathrm{~nm}$ (the difference between the FSRs of rings), resulting in $2.3 \mathrm{~nm}$ overall shift of the reflection peak from $\lambda=1535 \mathrm{~nm}$ to $\lambda=1537.3 \mathrm{~nm}$. Bleaching ring 1 for additional 45 seconds shifted the reflection peak additional $2.3 \mathrm{~nm}$ to $\lambda=1539.6 \mathrm{~nm}$.

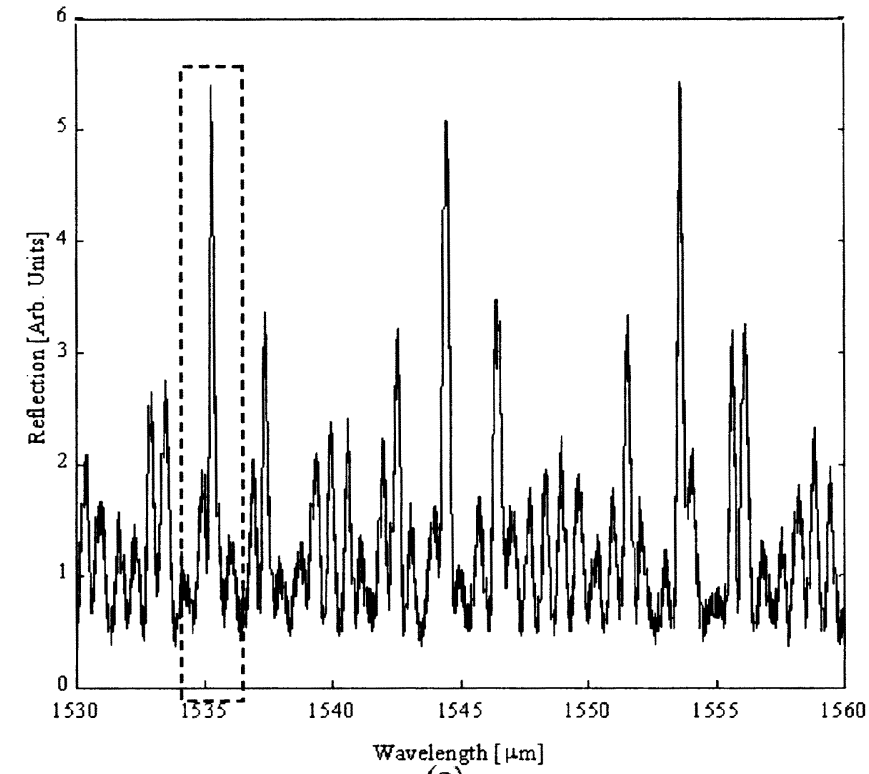

(a)

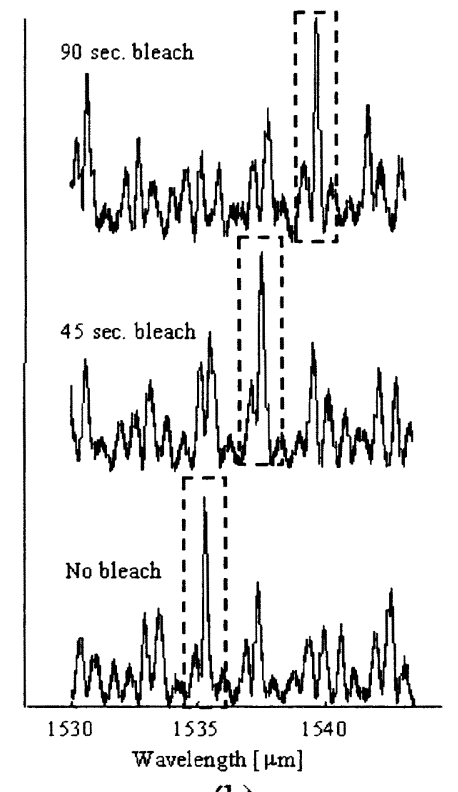

(b)

Fig. 3. (a) Measured reflected spectrum at port 1 and (b) tuning the reflection peak over more than $5 \mathrm{~nm}$.

\section{Conclusions}

We proposed and demonstrated a wavelength-selective reflector based on coupled microring resonators. The versatility of this class of reflectors lies in the fact that multiple rings can be used to attain narrow singlewavelength reflection or higher-order response which is ideal for use as laser end-mirrors. The usage of chromophore-doped polymer for the waveguides allows for simple and wide-range tuning of the microrings' resonance wavelengths and, correspondingly, the reflected wavelength either by photo-bleaching the chromophore or by the electro-optic effect. The employment of the Vernier effect enhances significantly (x5) the ability to tune the reflection peaks of the device.

\section{References}

[1] G. P. Agrawal, Fiber-optic communication systems. New York: Wiley, 1977

[2] A. Yariv, Optical electronics in modern communications. New York:Wiley, 1997

[3] T. Schrans, S. Sanders, and A. Yariv, "Broad-band wavelength tunable picosecond pulses from CW passively mode-locked twosection multiple quantum-well lasers," IEEE Photon. Technol. Lett., vol. 4, no. 4, pp. 323-326, 1992.

[4] J.-I. Hashimoto, T. Takagi, T. Kato, G. Sasaki, M. Shigehara, K. Murashima, M. Shiozaki, and T. Iwashima. "Fiber-Bragg-grating external cavity semicondutor laser (FGL) module for DWDM," IEEE J. of Lightwave Technol., vol. 21, no. 9, pp. 2002-2009, 2003.

[5] J. K. S. Poon, J. Scheuer and A. Yariv, "Wavelength-Selective Reflector Based on a Circular Array of Coupled Microring Resonators", IEEE Photon. Technol. Lett., vol. 16, no. 5, pp. 1331-1333, 2004.

[6] Y. Huang, G. T. Paloczi, J. Scheuer and A. Yariv, "Soft lithography replication of polymeric microring optical resonators", Opt. Express, vol. 11, no. 20, pp. 2452-2458, 2003.

[7] G. T. Paloczi, Y. Huang, J. Scheuer and A. Yariv, "Soft Lithography Molding of Polymer Integrated Optical Devices: Reduction of the Background Residue", J. Vac. Sci. Technol. B. vol. 22, no. 4, pp. 1764-1769, 2004.

[8] C. Zhang, L. R. Dalton, M.-C. Oh, H. Zahng and W. H. Steir, "'Low V $\pi$ Electrooptic Modulators from CLD-1: Chromophore Design and Synthesis, Material Processing, and Characterization", Chem. Mater., vol. 13, no. 9, pp. 3043-3050, 2001. 